\title{
lons and signal transduction in the macula densa
}

\author{
Scott C. Thomson and Roland C. Blantz \\ University of California and VAMC, San Diego, California, USA \\ Address correspondence to: Scott Thomson, Division of Nephrology/Hypertension, University of California \\ and VAMC, 3350 La Jolla Village Drive, San Diego, California 92161-9151, USA. \\ Phone: (858) 552-8585; Fax: (858) 552-7549; E-mail: sthomson@ucsd.edu.
}

See related article,

pages 681-688.

The body's internal environment is regulated, in large part, by processes occurring in the juxtaglomerular apparatus (JGA) of nephrons. One such processes is the well described phenomenon of tubuloglomerular feedback (TGF). A second and related process, the control of renin secretion by the macula densa, is the subject of the report by Cheng et al. (1) in the present issue of the JCI. Because TGF and the macula densa control of renin are intertwined, this research also bears on events in the JGA beyond its role in the systemic renin-angiotensin-aldosterone system (RAAS), which itself has profound effects on salt excretion. Products of the RAAS formed downstream from renin act to conserve salt by causing glomerular vasoconstriction and by increasing reabsorption of salt and water from the proximal and distal nephrons.

TGF allows changes in the chloride content of tubular fluid passing the macula densa at the end of Henle's loop to effect a reciprocal change in single nephron glomerular filtration rate (SNGFR). This negative feedback mechanism operates on a time scale of seconds and stabilizes both SNGFR and the rate at which salt reaches the distal nephron. In addition to initiating the TGF response, chloride also exerts an effect over longer times on renin secretion, the rate-limiting event in the RAAS. Specifically, when the apical macula densa is exposed to high chloride concentrations for 20 minutes or more, renin secretion from granular cells in the nearby afferent arteriole is suppressed (2). As a consequence, production of the peptide angiotensin II declines, as does the rate of salt and water reabsorption from the proximal tubule. The subsequent increase in late peroximal flow leads directly to an increase in chloride concentration at the macula densa (a consequence of the kinetics of salt transport in the waterimpermeable ascending loop of Henle). Therefore, suppression of RAAS activity at the nephron level will increase both late proximal flow and macula densa chloride.

\section{Positive and negative feedback in the control of salt excretion}

Unlike TGF, which stabilizes nephron function through negative feedback, the macula densa control of renin involves positive feedback. Thus, suppression of the RAAS by increased macula densa chloride should increase SNGFR, reduce proximal reabsorption, and beget a further increase in macula densa chloride. Positive feedback is uncommon in physiology and is inherently destabilizing. Furthermore, because of the well established role of the RAAS in maintaining extracellular volume and blood pressure through negative feedback (3), this effect of macula densa chloride on renin secretion cannot dominate the long-term control of either the RAAS or salt excretion.

Nonetheless, in teleological terms, the macula densa control of renin is not without potential utility. For exam- ple, incorporating a limited positive feedback effect on the amount of salt delivered to the distal nephron shortens the time required to come into salt balance after a change in salt intake, thereby lessening the impact of dietary salt on blood pressure $(3,4)$. Suppression of renin secretion by macula densa chloride may also provide a mechanism whereby TGF adapts to accommodate simultaneous increases in SNGFR and distal delivery. Because of TGF, chloride levels at the macula densa are inversely related to SNGFR, as shown in Figure 1. Therefore, these two parameters can only increase or decrease in parallel if the TGF curve itself is reset. Events in which such resetting is required include normal growth, pregnancy, loss of renal mass, and chronic salt loading. In each of these settings, the general direction of TGF resetting is rightward (Figure 1). Conversely, a return of nephron function to normal after pregnancy or acclimating to decreased salt intake involves a leftward resetting of TGF. This resetting must occur not only in the correct direction, but also to the proper extent. For TGF to perform its stabilizing role after resetting, it is necessary that the new ambient tubular flow remain aligned with the steep portion of the new TGF curve (5). It appears that ultimate control over the extent of TGF resetting is maintained by the macula densa itself, rather than being primarily driven by changes in the systemic neuroendocrine milieu $(6,7)$.

\footnotetext{
Figure 1

Idealized representation of TGF. Movement from point A to point B along the solid TGF curve signifies activation of TGF, which occurs within a few seconds of an increase in macula densa chloride. Because the TGF curve has negative slope, SNGFR and macula densa chloride cannot change in the same direction unless the TGF curve also changes. The dashed curve represents a shift, or resetting, of the TGF curve. Rightward resetting has been observed during prolonged activation of TGF. Since prolonged activation of TGF also suppresses renin, it is proposed that the rightward resetting is mediated in part by macula densa-dependent suppression of renin release. Cheng et al. (1) argue that the macula densa control of renin involves a pathway from low chloride to p38 MAP kinase to expression of COX-2.
}

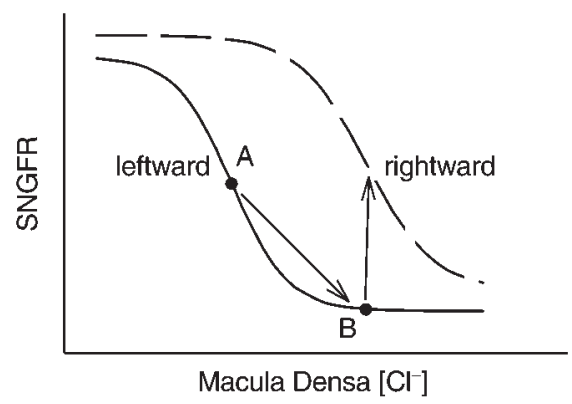

The Journal of Clinical Investigation | September 2000 | Volume 106 | Number 5 


\section{p38 and COX-2 and the resetting of TGF}

An increase in macula densa chloride induces a TGF response within seconds, but when this same stimulus is applied continuously for 30-60 minutes, a rightward shift in the TGF curve ensues. The mechanism whereby this shift in TGF comes about is not fully understood, but stimulation of macula densa nitric oxide synthase (NOS) and suppression of renin by macula densa chloride are two likely candidates. Until now, only the former effect has been implicated directly in this response. Inhibiting macula densa NOS interferes with changes in TGF that normally accompany salt loading in an animal (8) or during increased tubular flow in a single nephron (9). On the other hand, current evidence that renin suppression by the macula densa is involved with physiologic TGF resetting remains circumstantial. This evidence may be summarized as follows: Salt loading, which resets the TGF response in the macula densa, also suppresses COX-2 expression in this tissue (10). COX-2 is required for the production of prostaglandins, which are known renin secretagogues (11). Furthermore, COX-2 contributes to macula densa-mediated stimulation of renin release in response to perfusion with low chloride (12). Salt loading suppresses the RAAS, and the modification of the TGF response induced in saltloaded animals can be reversed by providing exogenous angiotensin II, which mediates many of the effects of renin (13). While these findings are consistent with the model, no evidence has yet been published showing directly that macula densa-dependent suppression of renin causes TGF resetting.

Cheng et al. (1) now add to this body of circumstantial evidence by elucidating a major step in the path whereby extracellular chloride governs COX-2 expression. Working with cells cultured from the thick ascending limb (cTAL), which bear apical transporters similar to those in the macula densa, these authors show that reducing ion flux into the cell via the $\mathrm{Na}-\mathrm{K}-2 \mathrm{Cl}$ transporter leads to increased phosphorylation of p38 mitogen-activated protein (MAP) kinase, which leads to increased transcription of COX-2. They further show that, in rats fed a low-salt diet, phosphorylated p38 is more abundant in and around the macula densa where it cosegregates with COX-2, again consistent with a role for p38 in COX-2 induction and, therefore, indirectly, in the suppression of renin release and the resetting of the TGF response.

Is the time course of COX-2 activation by this pathway consistent with this role? If macula densa control of renin mediates TGF resetting, then reducing extracellular chloride to stimulate COX2 is analogous to causing a leftward shift in TGF by imposing a sustained reduction in macula densa chloride, the reverse of the events depicted in Figure 1. Cheng et al. find p38 and COX-2 to be maximum after approximately 6 hours of low chloride, considerably longer than the 30-60 minutes required to reduce renin and to induce rightward resetting of TGF after increasing macula densa chloride $(2,6)$. However, it is not certain that rightward and leftward resetting must occur over the same period, and the authors did not test the time required to suppress COX-2 after changing from low to high chloride. The leftward shift in response to reduced chloride apparently requires activation of genes and translation of proteins, which may not be necessary for the response to increased chloride, allowing the latter process to occur more rapidly. In fact, we have tested the effect on TGF of a sustained reduction in macula densa chloride. This was done by first applying a proximal tubular diuretic to increase macula densa chloride for 24 hours and then withdrawing the diuretic. We found that TGF remained shifted to the right for many hours after the diuresis had subsided (9). The slow time course of the present experiments is consistent with those observations made in vivo.

The inverse relationship between prostaglandin formation and extracellular chloride is not unique to COX-2 in the loop of Henle: Cultured mesangial cells, which employ COX-1 in prostaglandin synthesis, are also subject to the suppressive effects of this anion, a finding that provides the basis of one hypothesis to explain the TGF effector mechanism. This hypothesis holds that changes in chloride at the apical macula densa are amplified in the basolateral interstium, where chloride inhibits angiotensin II-stimulated production of vasodilatory prostanoids by mesangial cells. These prostanoids effect TGF by acting in paracrine fashion on the glomerular arterioles (14-16). However, since TGF can change SNGFR within seconds in either direction, the mechanism whereby chloride affects COX-1 in mesangial cells must be different from the pathway described by Cheng et al. (1).

\section{Unanswered questions}

One important issue that remains unanswered by the present report concerns the mechanism whereby increased apical ion flux influences p38 MAP kinase in the macula densa or in cTAL cells. As pointed out by Cheng et al. (1), clues to this mechanism may lie in the factors that influence p38 phosphorylation in other systems. Because of the influence of chloride, it is tempting to speculate that changes in intracellular ion concentrations, per se, are responsible. However, intracellular ion contents differ markedly between macula densa cells and other cells of the thick ascending limb that express COX-2 at similar levels. For example, the macula densa cell contains much larger amounts of sodium and chloride (17), due to higher affinity of the apical $\mathrm{Na}-\mathrm{K}-2 \mathrm{Cl}$ transporter (18) and lesser basolateral Na-K-ATPase activity (19). Hence, regulatory mechanisms specific to the macula densa may be required for activation of $\mathrm{p} 38$. Indeed, recent data suggest that macula densa cells detect variations in lumenal $\mathrm{NaCl}$ through a signaling pathway involving the $\mathrm{Na}-\mathrm{K}-2 \mathrm{Cl}$ cotransporter in their apical surface. Peti-Peterdi and Bell (20) have proposed that ion flux mediated by this cotransporter leads to basolateral membrane depolarization via chloride channels and to calcium entry through voltage-gated calcium channels. As information comes to light regarding molecular events in the JGA, it will be important to consider what are the implications of these events for the control of organ function and maintenance of the body fluid compartments.

\section{Acknowledgments}

This work was done with funds provided by the Department of Veterans Affairs Research Service and by the NIH (National Institute of Diabetes and Digestive and Kidney Diseases, DK-28602).

\footnotetext{
1. Cheng, H.-F., Wang, J.-L., Zhang, M.-Z., McKanna, J.A., and Harris, R.C. 2000. Extracellular chloride regulates renal cortical cyclooxygenase-2 (COX-2) expression: role of $\mathrm{p} 38$. J. Clin. Invest. 106:681-688.

2. Lorenz, J.N., Weihprecht, H., Schnermann, J., Skott, O., and Briggs, J.P. 1991. Renin release from isolated juxtaglomerular apparatus depends on macula densa chloride transport. Am.
} 
J. Physiol. 260:F486-F493.

3. Guyton, A.C., Hall, J.E., Coleman, T.G., and Manning, R.D. 1990. The dominant role of the kidneys in the long term regulation of arterial pressure in normal and hypertensive states. In Hypertension, pathophysiogy, diagnosis, and management. B.M. Brenner and J. Laragh, editors. Raven Press. New York, New York, USA. 1029-1052.

4. Walser, M. 1985. Phenomenological analysis of electrolyte and water homeostasis. In The kidney, physiology and pathophysiology. D.W. Seldin and G. Giebisch, editors. Raven Press. New York, New York, USA. 3-13.

5. Thomson, S.C., and Blantz, R.C. 1993. Homeostatic efficiency of tubuloglomerular feedback in hydropenia, euvolemia, an acute volume expansion. Am. J. Physiol. 264:F930-F936.

6. Thomson, S.C., Blantz, R.C., and Vallon, V. 1996. Increased tubular flow induces resetting of tubuloglomerular feedback in euvolemic rats. Am. J. Physiol. 270:F461-F468.

7. Thomson, S.C., Vallon, V., and Blantz, R.C. 1997. Reduced proximal reabsorption resets tubuloglomerular feedback in euvolemic rats. Am. J.
Physiol. 273:R1414-R1420.

8. Welch, W.J., and Wilcox, C.S. 1997. Role of nitric oxide in tubuloglomerular feedback: effects of dietary salt. Clin. Exp. Pharmacol. Physiol. 24:582-586.

9. Thomson, S.C., et al. 1999. Temporal adjustment of the juxtaglomerular apparatus during sustained inhibition of proximal reabsorption. $J$. Clin. Invest. 104:1149-1158.

10. Harris, R.C., et al. 1994. Cyclooxygenase-2 is associated with the macula densa of rat kidney and increases with salt restriction. J. Clin. Invest. 94:2504-2510

11. Whorton, A., et al. 1977. Prostaglandins and renin release I. Stimulation of renin release from rabbit renal cortical slices by PGI2. Prostaglandins. 14:1095-1104.

12. Traynor, T.R., Smart, A., Briggs, J.P., and Schnermann, J. 1999. Inhibition of macula densa-stimulated renin secretion by pharmacological blockade of cyclooxygenase-2. Am. J. Physiol. 227:F706-F710.

13. Schnermann, J., and Briggs, J. 1984. Restoration of tubuloglomerular feedback in volume-expand- ed rats by angiotensin II. Am. J. Physiol 247:F246-F251.

14. Wilcox, C.S. 1983. Regulation of renal blood flow by plasma chloride. J. Clin. Invest. 21:726-735.

15. Podjarny, E., et al. 1986. Effect of angiotensin II on prostanoid synthesis in isolated rat glomeruli. Clin. Sci. 70:527-530.

16. Okuda, T., Yamishita, N., and Kurokawa, K. 1986 Angiotensin II and vasopressin stimulate calcium-activated chloride conductance in rat mesangial cells. J. Clin. Invest. 78:1443-1448.

17. Lapointe, J.Y., Laamarti, A., and Bell, P.D. 1998. Ionic transport in macula densa cells. Kidney Int. 67(Suppl.):S58-S64.

18. Laamarti, M.A., Bell, P.D., and Lapointe, J.Y. 1998. Transport and regulatory properties of the apical $\mathrm{Na}-\mathrm{K}-2 \mathrm{Cl}$ cotransporter of macula densa cells. Am. J. Physiol. 275:F703-F709.

19. Schnermann, J., and Marver, D. 1986. ATPase activity in macula densa cells of the rabbit kidney. Pflugers Arch. 407:82-86.

20. Peti-Peterdi, J., and Bell, P.D. 1999. Cytosolic $[\mathrm{Ca} 2+]$ signaling pathway in macula densa cells. Am. J. Physiol. 277:F472-F476. 